\title{
ABUNDANCES OF HEAVY ELEMENTS IN THE MAGELLANIC CLOUDS
}

\author{
Stephen C. Russell, Michael S. Bessell and Michael A. Dopita \\ Mount Stromlo and Siding Spring Observatories \\ Private Bag PO Woden ACT 2611 \\ Australia
}

\begin{abstract}
This paper provides the first reliable determination of the iron abundance of the Magellanic clouds. There is clear evidence for carbon and nitrogen depletion, with respect to iron, in the clouds which can be explained by the relative youth of the clouds compared to our own Galaxy. Oxygen depletion relative to iron is also in evidence and it is suggested here that this is due to preferential loss of ejecta from high mass stars. Finally, our discovery of an extremely low abundance star in the SMC, coupled with the identification of a similar star by spite et al. (1987), calls in to question whether or not the SMC is indeed a well mixed system as previously assumed.
\end{abstract}

\section{INTRODUCTION}

This paper presents a preliminary report on a part of a complete survey of elements in the Magellanic Clouds. Traditionally, abundances in the clouds have been derived from spectra of HII regions for the lighter elements, and from spectra of supergiants for the heavier elements. The supergiants selected are young objects with atmospheric abundances little changed from their initial values. Unfortunately previous efforts at determining these heavy element abundances have relied on high dispersion observations of the brightest, most extreme stars (for example Przybylski 1968, 1971, 1972; Wolf 1972, 1973). These studies have resulted in abundance estimates for the same star by different authors that differ by up to 0.7 dex. The problem with these stars is that physical parameters are difficult to determine due to their temperature and a break down in the classically held assumptions of Local Thermodynamic Equilibrium (LTE) and radiative equilibrium.

\section{DISCUSSION}

In order to avoid the problems noted above, we chose stars with the lowest luminosity and highest gravity possible. The need for a plentiful supply of lines in the spectra while still allowing adequate continuum placement, and the requirement that molecular opacity remains unimportant, further restricted our choice to spectral classes between 
F0 and F8. Since the Magellanic Clouds were both expected to be well mixed (see Pagel et al 1978), only a small number of stars were required to be observed in order to obtain the global abundances of the clouds. This paper gives the preliminary results from 5 stars in the SMC and 6 in the LMC, the latter of which have only iron and titanium abundance measures so far.

Estimates of the effective temperatures and gravities of the program stars were made using 4-color and and $\mathrm{H} \beta$ photometry (Russell et al 1987). Spectra were then obtained using the $5 \mathrm{~m}$ Anglo-Australian Telescope (AAT), and the $2.3 \mathrm{~m}$ telescope at sSO with dispersions in the range of 3 to $5 \mathrm{Amm}^{-1}$. The equivalent widths of the lines were measured and the abundances derived with the Kurucz program WIDTH6. Fine analyses were run using models with different surface gravity and effective temperature (interpolated from the grid of Kurucz (1979)), and different values for microturbulence, until abundances were derived independent of line strength, ionization state and excitation potential.

The first interesting result from the analysis concerned the physical properties of the stars themselves. It seemed that there was a systematic difference between the microturbulent velocities measured for the stars we observed in the two clouds. The range of velocities for the SMC were between 4 and $5 \mathrm{kms}^{-1}$, while for the IMC they were between 7 and $12 \mathrm{kms}^{-1}$. Although both ranges were well within those found for supergiants in the Galaxy, their lack of overlap with each other may suggest that metallicity affects a star's microturbulent velocity.

The results of the abundance analysis are summarized in Table 1, where our values represent deficiencies with respect to Canopus as measured by us using the same wavelength intervals and the same method of reduction. Our values for $[\mathrm{Fe} / \mathrm{H}]$ in the SMC are somewhat higher than estimates made by other authors from photometry and low resolution observations of the calcium $\mathrm{H}$ and $\mathrm{K}$ lines, although still within their error bars, while our detailed abundances are, in general, somewhat lower than those estimated by Foy (1981) and Thévenin and Foy (1986) from medium resolution observations of $\mathrm{GOIa}$ stars. These latter stars are however, of high luminosity and low gravity, and considering the relatively low resolution observations made on them, their results may be somewhat less reliable than our own. In the LMC, on the other hand, our results agree fairly well with those from other authors, especially the most relevant and accurate results of van Genderen et al (1985). These results then, represent the first reliable, direct measure of the iron abundance in the clouds. From this basis is possible to say much concerning the abundances of other elements relative to iron as compared to the same ratios in the Galaxy.

Figure 1 show the depletion of all the elements measured in the literature (see Dopita 1987 for a summary) for the Clouds, where values from this study (Table 1) have been used whenever possible. The values for the depletion of iron as measured by us, have been marked with dashed lines since, in general, elements in our own galaxy tend to follow the deficiency of iron. The most striking feature is the underabundance of carbon and nitrogen with respect to iron in both the clouds. The variation of the carbon to iron ratio against the iron abundance in our own Galaxy see for example (Twarog and Wheeler 1982), 
is close to being constant. This would indicate that both elements are derived from similar mass stars. However, comparison with the results for the clouds shows that there is a drastic underabundance of carbon relative to iron in the clouds. This would tend to indicate that in fact iron is produced by somewhat higher mass stars than those producing carbon, and the latter stars have not yet had time to enrich their local Interstellar Medium (ISM) to equilibrium proportions in the Clouds. Peimbert (1987) came to a similar conclusion in an effort to explain the difference between the ${ }^{12} \mathrm{C} /{ }^{13} \mathrm{C}$ ratio in the sun and in the Galactic ISM. He suggested that a substantial fraction of the ${ }^{12} \mathrm{C}$ is produced by intermediate mass stars in the $M_{0} M_{0} \leq 2$ range, and therefore the instantaneous recycling approximation is not valid. Similar deficiencies are obtained for nitrogen (see Twarog and Wheeler 1982), so although the bulk of both nitrogen and carbon are produced by similar mass stars, iron must come from the higher mass stars.

Oxygen production in the Galaxy, see for example (Matteucci, 1986), is on the other hand, far from being a linear relation with iron. The constant $[0 / \mathrm{Fe}]$ ratio below an iron abundance of $[\mathrm{Fe} / \mathrm{H}]=-1.0$ is interpreted as being due to production of both oxygen and iron (to a limited extent) by high mass star supernovae (Type II). However, the change in slope beyond an iron abundance of -1.0 is due to the bulk of iron being produced by the medium mass stars, with the appropriate time lag. Plotting the values for the SMC and LMC on the same diagram reveals a large underabundance of oxygen with respect to iron as compared to the Galaxy. Any chance of explaining this by invoking a different Initial Mass Function (IMF) for low metallicity environments, is largely ruled out due to the effect that this would have on, for instance, the G-dwarf problem. Indeed Larson (1986) was tempted to suggest that a double peaked IMF with a steeply declining Star Formation Rate (SFR), would provide an explanation for the G-dwarf problem while accounting for the missing dark matter with the resulting large density of white dwarfs. Unfortunately this scenario would result in yet further enrichment in oxygen relative to iron in the Magellanic Clouds.

One possible explanation is that oxygen is preferentially lost, relative to iron, from the clouds, while being retained in the Galaxy. A possible scenario for this is suggested by observations of supergiant HI loops in the Clouds (see for example Dopita, Mathewson and Ford 1985). These are vast loops of HI gas, on scales of several kiloparsecs, observed to be expanding at velocities of the order of $35 \mathrm{kms}^{-1}$, and thought to be powered by supernovae explosions from contagious massive star formation. They eject material into the hot haloes of their parent galaxies as efficiently in the case of the clouds as in the case of the Galaxy (Dopita 1985), but each galaxy can only retain the material according to the strength of its gravitational field. Iron, on the other hand, is produced much later by lower mass stars which are unable to provide the necessary combined energy to blast a hole in the ISM through to the hot galactic halo. Moreover, the readymade holes from the high mass stars dissipate in the order of $10^{7}$ years, long before the stars responsible for iron production end their lives. Thus we conclude that large amounts of oxygen escape preferentially from smaller systems, which are nevertheless able to hang on to the majority of their iron. 
TABIT, 1

\section{Macellanic cloud star Abundances}

SMC

IMC

ELEMENT

$\begin{array}{ccccc}{[M / H]} & \text { No.of } & {[M / H]^{1}} & {[M / H]} & \text { No.of } \\ (4 F \text { Ib) } & \text { Lines } & (F, T F) & (6 F \text { Ia-b) } & \text { Lines }\end{array}$

\begin{tabular}{|c|c|c|c|c|c|c|c|c|}
\hline Mg & -0.61 & \pm 0.26 & 2 & -0.2 & & & & \\
\hline $\mathrm{Ca}$ & -0.33 & \pm 0.24 & 2 & -0.5 & & & & \\
\hline Sc & -1.04 & \pm 0.13 & 1 & -0.3 & & & & \\
\hline $\mathbf{T i}$ & -0.52 & \pm 0.07 & 20 & -0.35 & $-0.22 \pm 0.14$ & 18 & - & 101 \\
\hline $\mathrm{Cr}$ & -0.49 & \pm 0.15 & 4 & -0.25 & & & & \\
\hline $\mathrm{Fe}$ & -0.55 & \pm 0.10 & 20 & $-0.4 \pm 0.2$ & $-0.18 \pm 0.19$ & 19 & - & 61 \\
\hline $\mathbf{Z r}$ & -0.43 & \pm 0.23 & 1 & -0.4 & & & & \\
\hline $\mathbf{B a}$ & -0.80 & \pm 0.18 & 1 & -0.4 & & & & \\
\hline $\mathrm{Ce}$ & -0.51 & \pm 0.22 & 1 & -0.6 & & & & \\
\hline
\end{tabular}

1 Foy (1981), Thévenin and Foy (1986)

Other estimates of the SMC metallicity: Smith, 1980: $-0.8 \pm 0.2$; Harris, 1981,83: $-0.65 \pm 0.4 ;$ Pel el al., 1981: $-0.7 \pm 0.25$

Other estimates of the IMC metallicity: Smith, 1980: $-0.30 \pm 0.2$ Harris, 1983: $-0.09 \pm 0.3$; van Genderen et al., 1985: $-0.18 \pm 0.02$
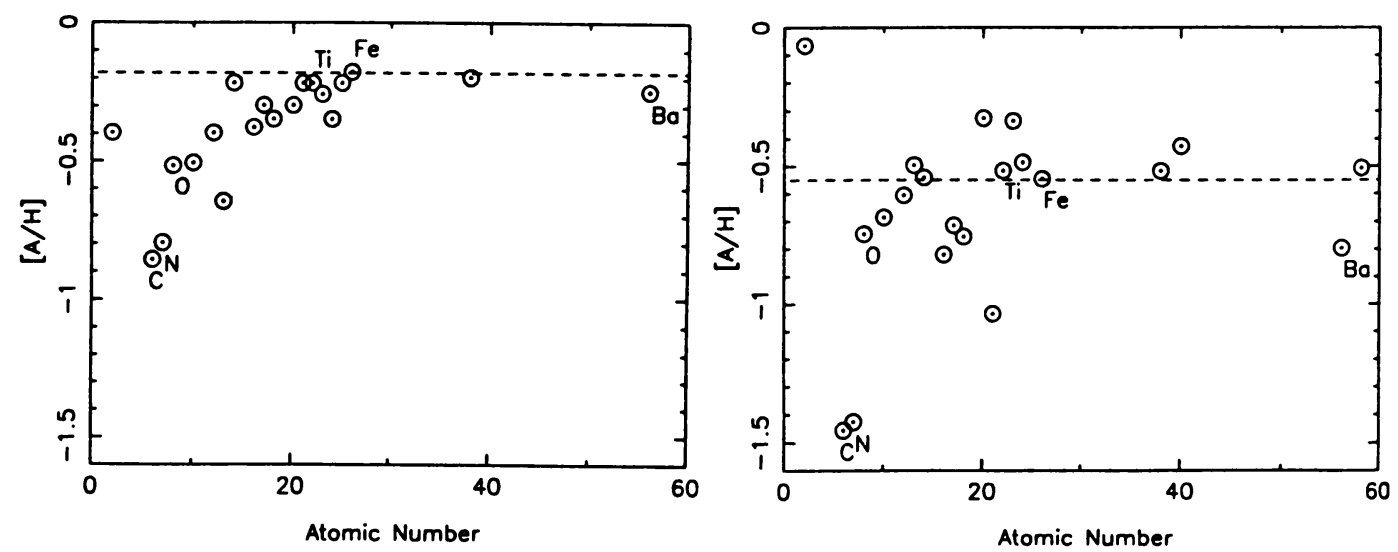

Figure 1. Abundances of the elements in the LMC and SMC relative to Canopus. The dashed lines represents the iron abundance. 
The general trend in abundance for the LMC (Figure 1a) is one of rapidly decreasing overdeficiency for the lighter elements until calcium, after which there is a normal deficiency. This is similar to, but not so steep as the enrichment expected from the carbon deflagration supernovae, as put forward by Nomoto et al. (1984, model W7). The results of Thielmann and Arnett (1985) for the expected contributions to the light elements by Type II supernovae suggest that these would tend to flatten out any trend in overdeficiencies produced by other means. The observed slope in overdeficiency therefore, supports the proposed loss to the system of elements ejected from high mass supernovae.

The SMC (Figure 1b), on the other hand, shows very little slope in overdeficiency. This may reflect the youth of the system, whereby there has been too little time for enrichment due to carbon deflagrating stars to have had much effect. Barium is one of the few elements known to be produced largely by the s-process and therefore its measurement is of special interest. Unfortunately, all of our measurements of its abundance depend upon one strong line, and as such they are very sensitive to the exact microturbulent velocity. The results for barium are therefore only tentative at present, and should be treated with caution until we measure more lines. The same problem is true for Scandium which our results show as being of rather low abundance for the SMC.

We must point out that one of the stars we observed in the SMC (Azzopardi and Vigneau 1975: No.79) has a much lower abundance ([Fe/H] $\approx$ $-1.0,[\mathrm{Ti} / \mathrm{H}] \approx-1.7)$ than the others. The iron abundance is similar to the deficiency $([\mathrm{Fe} / \mathrm{H}]=-1.2)$ measured by spite et al. (1986) for a star in the SMC cluster NGC 330. The latter star is in a very young globular cluster which has been suggested from other observations to be extremely metal deficient, thus to some extent adding weight to the findings of Spite et al. These stars tend to suggest that the SMC at least is not so well mixed as was previously thought.

\section{CONCLUSIONS}

We have measured the iron abundance in the Magellanic Clouds directly and reliably and found the following results:

LMC: $[\mathrm{Fe} / \mathrm{H}]=-0.18 \pm 0.19$

SMC: $[\mathrm{Fe} / \mathrm{H}]=-0.55 \pm 0.10$

Combining these results with those derived from the literature we are able to say that both carbon and nitrogen are deficient with respect to iron in the clouds. This is probably due to the youth of the clouds and the fact that the these elements are produced mainly by somewhat lower mass stars than those responsible for the bulk of the iron production. oxygen is underabundant with respect to iron in the clouds relative to our own galaxy. We suggest that this is due to the preferential loss of ejecta from high mass stars in low mass galaxies. Finally, we have identified a very metal deficient star, which together with one reported by spite et al.(1986), suggest that the SMC is not so well mixed as previously thought. 


\section{REFERENCES}

Azzopardi, M. and Vigneau, J. 1975 Astr. Astrophys. Suppl, 22,285.

Clegg, R.E.S., Lambert, D.L. and Tomkin, J. 1981 Astrophys.J., 250, 262. Dopita, M.A. 1985, in 'Birth and Evolution of Massive Stars and Stellar Groups', ed. by W.Boland and H. van Woerden, p. 269.

Dopita, M.A. 1987, in 'The Magellanic System', ed. by M.A.Dopita, Princeton U.P., in preparation.

Dopita, M.A., Mathewson, D.S. and Ford, V.L.1985 Astrophys. J.,297, 599. Foy, R. 1981 Astr. Astrophys., 103, 135.

Harris, H.C. 1981 Astr. J., 86, 1192.

Harris, H.C. 1983 Astr. J., 88, 507.

Kurucz R.L. 1979 Astrophys. J., 40, 1.

Larson R. 1986 Mon. Not R. Astr. Soc., 218, 409.

Matteucci, F. 1986 Publ. Astr. Soc. Pac., 98, 173.

Nissen, P.E., Edvardsson, B. and Gustafsson, B. 1985 'ESO Workshop

on Production and Distribution of $C, N, O$ Elements', I.J. Danziger,

F. Matteucci and $\mathrm{K} . \mathrm{Kj}$ (eds.) (ESO Publication), p. 131.

Nomoto, K., Thielemann, F.-K. and Yokoi, K. 1984 Astrophys.J., 286, 644.

Pagel, B.E.J., Edmunds, M.G., Fosbury, R.A.E. and Webster, B.L. 1978, Mon. Not. R. Astr. Soc., 184, 569.

Peimbert, M. 1987, in 'Star Forming Regions', IAU Symposium No. 115, M. Peimbert and J. Jugaku (eds.) (Dordrecht: Reidel), p. 111.

Pel, J.W., van Genderen, A.M. and Lub, J. 1981 Astr. Astrophys., 99, L1. Przybylski, A. 1968 Mon. Not. R. Astr. Soc., 139, 313.

Przybylski, A. 1971 Mon. Not. R. Astr. Soc., 152, 197.

Przybylski, A. 1972 Mon. Not. R. Astr. Soc., 159, 155.

Russell.S.C., Bessell, M.S and Dopita, M.A.1987, Proc.Astr.Soc.Aust., in press.

Smith H.A.1980 Astr.J., 85, 848.

Sneden, C., Lambert, D.L. and Whitaker, R.W.1979 Astrophys.J., 234, 964.

Spite, M., Cayrel, R., Francois, P., Richtler, T. and Spite, F.1987, preprint.

Spite, M. and Spite, F. 1978 Astr. Astrophys., 67, 23.

Thévenin, F. and Foy, R. 1986 Astr. Astrophys., 155, 145.

Thielemann, F.-K. and Arnett, W.D. 1985, in 'Nucleosynthesis, Challenges and New Developments', W.D.Arnett and J.W.Truran (eds.) (University of Chicago Press), p. 151.

Twarog, B.A. and Wheeler, J.C. 1982 Astrophys. J., 261, 636.

van Genderen, van Driel W.and Greidanus, H.1985 Astr.Astrophys.,155, 72.

Wolf, B. 1972 Astr. Astrophys., 20, 275.

Wolf, B. 1973 Astr. Astrophys., 28, 335.

\section{DIScussion}

SNEDEN How do the microturbulent velocities that were derived for Ix stars compare with those determined for stars of similar spectral type in oux galaxy?

BESSELL The range of microturbulent velocities in the rmC stars is similar to that in the $F$ supergiants analysed by Osmer. However, the gravity of the galactic supergiants are more difficult to assess for correlation of microturbulence with $\log \mathrm{g}$. 